\title{
SOLUTIONS DE VISCOSITE DES EQUATIONS DE HAMILTON-JACOBI EN DIMENSION INFINIE. CAS STATIONNAIRE
}

\author{
El Mahjoub El Haddad
}

\begin{abstract}
We show the uniqueness and the existence of viscosity solutions of Hamilton-Jacobi equations on a smooth Banach spaces. The tool used is the variational principle of Deville, Godefroy and Zizler. The existence is given by Perron's method. So we give a comparaison assertion for semicontinuous solutions.
\end{abstract}

\section{Introduction}

Dans ce travail nous étudierons l'existence et l'unicité des "solutions de viscosité" des équations de Hamilton-Jacobi en dimension infinie. Nous considérons le problème stationnaire:

$$
u(x)+H(x, D u(x))=0 \operatorname{sur} X,
$$

où $X$ est un espace de Banach, $H(x, p)$ est une fonction réelle uniformément continue, définie sur $X \times X^{*}, u(x)$ est la fonction réelle inconnue, $D u$ désigne la dérivée au sens de Fréchet de $u$ par rapport à $x$, et $X^{*}$ est l'espace dual de $X$.

Cette notion de solution a été introduite par M. G. Crandall et P. L. Lions (cf. [5] et [6]), où ils ont donné la théorie de base des équations de Hamilton-Jacobi du premier ordre en dimension finie en démontrant l'existence et l'unicité de solutions de viscosité de (1.1) avec l'hypothèse " $H$ continue sur $\mathbb{R}^{n} \times \mathbb{R}^{n}$ ". Un an plus tard M. G. Crandall, L. C. Evans et P. L. Lions ont reformulé et simplifié ce travail (cf. [4]). Après, plusieurs auteurs se sont intéressés à ce sujet, en particulier H. Ishii ([16], $[\mathbf{1 7}],[\mathbf{1 8}],[\mathbf{2 0}])$, Barles [1], I. Capuzzo Dolcetta [3] et d'autres. Quelques années plus tard, M. G. Crandall et P. L. Lions ont remarqué que ces équations peuvent être étudiées aussi en dimension infinie. En fait, en dimension finie, pour comparer les sous-solutions aux sur-solutions 
de viscosité, l'astuce était de maximiser après pénalisation une fonction semi-continue supérieurement dite fonction auxiliaire. Ainsi, ils ont supposé des hypothèses techniques d'uniformité sur le Hamiltonien, sur les sous-solutions et les sur-solutions de viscosité, ce qui a été justifier par l'utilisation des jeux différentiels pour l'existence. Mais ils se sont intéressés aux espaces de Banach qui possèdent la propriété de RadonNikodym et une fonction bosse régulière, car sur ces espaces, il y a le principe variationnel de Stegall (cf. [21]). Ce principe variationnel leur a permis de donner une série de travaux (cf. [7]).

Plus récemment, R. Deville, G. Godefroy et V. Zizler ont trouvé un principe variationnel (cf. [11]) qui a étendu celui de J. M. Borwein et D. Preiss (cf. [2]) par une démonstration simple en utilisant le théorème de Baire. Ce résultat leur a donné l'idée d'étudier les équations de Hamilton-Jacobi sur des espaces plus vastes que ceux de M. G. Crandall et $\mathrm{P}$. L. Lions, c'est à dire des espaces qui possèdent une fonction bosse de classe $\mathcal{C}^{1}$ et Lipschitzienne. Ils ont obtenu à la fois l'existence et l'unicité des solutions de viscosité bornées du problème stationnaire de la forme:

$$
u(x)+H(D u(x))=f(x) \text { sur } X,
$$

où $X$ est un espace de Banach possédant une fonction bosse de classe $\mathcal{C}^{1}$ et Lipschitzienne, $H: X^{*} \longrightarrow \mathbb{R}$ une fonction uniformément continue et $f$ une fonction réelle, bornée et uniformément continue sur $X$. Pour l'existence, ils ont utilisé la méthode de Perron introduite par H. Ishii en 1987 (cf. [19]). Cette méthode est efficace si on arrive à comparer une sous-solution semi-continue supérieurement à une sur-solution semicontinue inférieurement.

Voici maintenant le plan de ce travail: dans la première section, nous rappelons la définition de solution de viscosité du problème (1.1), et nous donnons quelques préliminaires.

Dans la seconde section, nous montrons le résultat de comparaison dans le cas des sous-solutions majorées linéairement et des sur-solutions minorées linéairement, ce qui est une extension à la fois des travaux de R. Deville, G. Godefroy et V. Zizler (cf. [11]) à un cas non "borné" et de ceux de M. G. Crandall et P. L. Lions dans le cas stationnaire (cf. [7]), seulement ici, le problème est considéré sur l'espace tout entier au lieu d'un ouvert quelconque. La démonstration est indirecte puisqu'au début, on se place dans le cas où la sur-solution de viscosité est de classe $\mathcal{C}^{1}$. Pour se ramener à ce cas, on se place sur l'espace $X \times X$ et nous utilisons le fait qu'une fonction uniformément continue est Lipschitzienne pour les grandes distances (lemme 3.4). Nous construisons une sur-solution de viscosité d'un problème sur $X \times X$ de même forme que (1.1) avec un Hamiltonien formé par la différence de deux Hamil- 
toniens sur $X \times X^{*}$. Cette nouvelle sur-solution sera arbitrairement petite sur la diagonale. Ainsi le résultat découle immédiatement du fait que la différence d'une sous-solution et d'une sur-solution de viscosité sur $X \times X$ du problème (1.1) est une sous-solution de viscosité du problème construit sur $X \times X$. Nous donnerons aussi des exemples, pour montrer la nécessité de l'hypothèse géométrique et de la continuité uniforme de l'Hamiltonien. Dans la dernière section, nous verrons à l'aide de la méthode de Perron pour les solutions de viscosité, comment le théorème de comparaison (Théorème 3.1) peut donner l'existence des solutions de viscosité et leur régularité en fonction du module de continuité de l'Hamiltonien.

\section{Définitions et préliminaires}

Dans tout ce travail l'espace de Banach $X$ vérifie la condition géométrique suivante:

$$
\left\{\begin{array}{l}
\text { Il existe une fonction } b: X \longrightarrow \mathbb{R}, \text { de classe } \mathcal{C}^{1}, \\
\text { Lipschitzienne avec support borné et non vide. }
\end{array}\right.
$$

Une telle fonction est appelée fonction bosse régulière.

Ici dans la plupart des démonstrations nous utiliserons le principe variationnel de R. Deville, G. Godefroy et V. Zizler dont voici l'énoncé:

Théorème 2.1. Soient $X$ un espace de Banach, $f: X \longrightarrow \mathbb{R}$ une fonction semi-continue inférieurement et bornée inférieurement, telle que $D(f) \neq \emptyset$ et $\varepsilon>0$. Supposons qu'il existe une fonction bosse de classe $\mathcal{C}^{1}$ et Lipschitzienne sur $X$. Alors, il existe une fonction $g$ de classe $\mathcal{C}^{1}$ sur $X$, telle que:

a) $f+g$ a un minimum en un point $x_{0} \in D(f)$,

b) $\|g\|_{\infty}=\sup \{|g(x)| ; x \in X\}<\varepsilon$ et $\left\|g^{\prime}\right\|_{\infty}=\sup \left\{\left\|g^{\prime}(x)\right\|_{\infty} ; x \in\right.$ $X\}<\varepsilon$.

Rappelons que si l'espace de Banach $X$ admet une norme équivalente de classe $\mathcal{C}^{1}$ sur $X \backslash\{0\}$, alors l'hypothèse (A) est vérifiée. L'inverse n'est pas vrai en général (cf. [15] pour un contre-exemple). Cependant on peut produire une fonction régulière et homogène à partir d'une fonction bosse régulière. Ceci est donné par la proposition suivante qui est démontrée dans $[\mathbf{1 2}]$.

Proposition 2.2. Supposons que l'espace de Banach $X$ vérifie l'hypothèse (A). Alors il existe une fonction d Lipshitzienne sur $X$ telle que:

(i) d est de classe $\mathcal{C}^{1}$ sur $X \backslash\{0\}$ 
(ii) $d(t x)=|t| d(x)$ pour $x \in X$ et $t \in \mathbb{R}$

(iii) il existe une constante $K>0$ telle que

$$
\|x\| \leq d(x) \leq K\|x\| \text { pour } x \in X .
$$

Le problème (1.1) n'admet pas de solutions classiques en général (voir [12] pour des exemples). C'est pourquoi M. G. Crandall et P. L. Lions ont introduit la notion de solution de viscosité en utlisant les sousdifférentiels et les sur-différentiels de fonctions dont voici un rappel:

Pour $f: X \longrightarrow \mathbb{R} \cup\{+\infty\}$ une fonction semi-continue inférieurement, on note $D(f)$ le domaine de $f$ :

$$
D(f):=\{x \in X ; f(x)<+\infty\}
$$

et on définit:

$$
\begin{aligned}
D^{-} f(x)=\left\{\varphi^{\prime}(x) ; \varphi: X \longrightarrow\right. & \mathbb{R} \text { de classe } \mathcal{C}^{1} \\
& \text { et } f-\varphi \text { a un minimum local en } x\} .
\end{aligned}
$$

On dit que $f$ est sous-différentiable en $x$ si $D^{-} f(x) \neq \emptyset$ (pour $x \notin D(f)$, on définit $D^{-} f(x)=\emptyset$ ). De manière similaire, on peut définir le surdifférentiel de $f$ en $x$ :

$$
\begin{aligned}
D^{+} f(x)=\left\{\varphi^{\prime}(x) ; \varphi: X \longrightarrow\right. & \mathbb{R} \text { de classe } \mathcal{C}^{1} \\
& \text { et } f-\varphi \text { a un maximum local en } x\} .
\end{aligned}
$$

Définition 2.3. Une fonction $u: X \longrightarrow \mathbb{R}$ est une sous-solution de viscosité de (1.1) si:

i) $u$ est semi-continue supérieurement

ii) pour tout $x \in X$ et tout $p \in D^{+} u(x)$ :

$$
u(x)+H(x, p) \leq 0 .
$$

La fonction $u$ est une sur-solution de viscosité de (1.1) si:

iii) $u$ est semi-continue inférieurement

iv) pour tout $x \in X$ et tout $p \in D^{-} u(x)$ :

$$
u(x)+H(x, p) \geq 0 .
$$

Finalement $u$ est une solution de viscosité de (1.1) si $u$ est à la fois sous-solution et sur-solution de viscosité de (1.1). 


\section{Remarques.}

1) D'après cette définition, on voit que les solutions de viscosité sont seulement continues.

2) Si $X$ vérifie l'hypothèse $(\mathrm{A})$, alors les solutions classiques de (1.1) sont aussi des solutions de viscosité. En effet, si $u$ est une solution classique de (1.1), alors $u$ est de classe $\mathcal{C}^{1}$ sur $X$. Et en utilisant la Proposition VIII.1.2 [12], on trouve $D^{+} u(x)=D^{-} u(x)=\{D u(x)\}$ pour $x \in X$, ce qui montre que $u$ est solution de viscosité de (1.1).

3) Considérons dans $\mathbb{R}$ la fonction $u(x)=|x|$. Pour $x \neq 0$, on a $\left(u^{\prime}(x)\right)^{2}-1=0$, et il est facile de voir que $D^{+} u(0)=\emptyset$ et $D^{-} u(0)=$ $[-1,1]$, ce qui montre que $u$ est solution de viscosité de l'équation $1-$ $\left(u^{\prime}\right)^{2}=0$, mais qu'elle n'est pas solution de viscosité de $\left(u^{\prime}\right)^{2}-1=0$. On est donc confronté à un problème non linéaire.

\section{Unicité des solutions de viscosité}

Dans cette section nous montrons le théorème de comparaison suivant:

Théorème 3.1. Supposons que l'espace de Banach X satisfait l'hypothèse (A). Soient $H_{1}, H_{2}$ deux Hamiltoniens uniformément continues sur $X \times X^{*}$. Soient $u, v$ deux fonctions réelles définies sur $X$. Supposons qu'il existe une constante $C \geq 0$ telle que $u(x) \leq C(1+\|x\|)$ et $v(x) \geq$ $-C(1+\|x\|)$ pour $x \in X$.

Si u est une sous-solution de viscosité de

$$
u+H_{1}(x, D u)=0 \text { sur } X
$$

et $v$ est une sur-solution de viscosité de

$$
v+H_{2}(x, D v)=0 \text { sur } X
$$

alors pour tout $\varepsilon>0$, il existe $K_{\varepsilon} \geq 0$ tel que

$$
u(x)-v(y) \leq\left(\varepsilon^{2}+K_{\varepsilon}\|x-y\|^{2}\right)^{\frac{1}{2}}+\sup _{X \times X^{*}}\left(H_{1}-H_{2}\right)
$$

pour $x, y \in X$.

\section{Remarques.}

1) Dans le Théorème 3.1, si on prend $H_{1}=H_{2}=H$, on obtient pour tout $\varepsilon>0, u(x)-v(x) \leq \varepsilon$ pour $x \in X$. Donc $u \leq v$ sur $X$. Ceci montre que l'équation (1.1) a une unique solution de viscosité. 
2) Soient $X$ un espace de Hilbert et $H: X \times X$ un Hamiltonien défini par $H(x, p)=-\langle x, p\rangle(\langle$,$\rangle désigne le produit scalaire sur X)$.

Le Hamiltonien $H$ est uniformément continu sur les sous-ensembles bornés de $X^{2}$, mais il n'est pas uniformément continu sur $X^{2}$. Les fonctions $u=0$ et $v(x)=\|x\|$ sont des solutions de viscosité uniformément continues de $u+H(x, D u)=0$ sur $X$. Ceci montre que l'hypothèse d'uniforme continuité de le Hamiltonien $H$ dans le Théorème 3.1 ne peut être supprimée. D'autres conditions, plus générales (mais plus techniques) ont été considées par M. G. Crandall, P. L. Lions dans [7] et H. Ishii dans $[\mathbf{1 9}]$. Ces conditions plus générales sont motivés par les applications à des problèmes de contrôl optimal ou de jeux différentiels.

3) Ici on montre la nécessité de la condition géométrique $(\mathrm{A})$ dans le Théorème 3.1. Pour cela, on considère l'espace de Banach $X=\ell^{1}(\mathbb{N})$ et le Hamiltonien $H: \ell^{1}(\mathbb{N}) \times \ell^{\infty}(\mathbb{N}) \longrightarrow \mathbb{R}$ défini par $H(x, p)=-\left(\|p\|_{\infty}+\right.$ $\left.\|x\|_{1}\right)+1$ qui est uniformément continu. Maintenant, considérons les fonctions réelles $u$ et $v$ définies $\operatorname{sur} \ell^{1}(\mathbb{N})$ par $u(x)=\frac{1}{2}\left(\|x\|_{1}+1\right)$ et $v(x)=\|x\|_{1}$. On affirme que $u$ est une sous-solution de viscosité et $v$ est une sur-solution de viscosité du problème $u+H(x, D u)=0 \operatorname{sur} \ell^{1}(\mathbb{N})$. En effet, pour $x=\left(x_{n}\right) \in \ell^{1}(\mathbb{N}), D^{-} v(x)=\left\{p=\left(p_{n}\right) \in \ell^{\infty}(\mathbb{N}) ;\left|p_{n}\right| \leq 1\right.$ et $p_{n}=\operatorname{sign} x_{n}$ si $\left.x_{n} \neq 0\right\}$. Donc si $p \in D^{-} v(x), v(x)+H(x, p)=$ $\|x\|_{1}-\|p\|_{\infty}+1-\|x\|_{1}=1-\|p\|_{\infty} \geq 0$. Puisque $x \longrightarrow\|x\|_{1}$ est convexe et nulle part Fréchet différentiable sur $\ell^{1}(\mathbb{N})$, on a $D^{+} u(x)=\emptyset$ pour $x \in \ell^{1}(\mathbb{N})$. Mais $v(x)-u(x)=\frac{\|x\|_{1}-1}{2}$; par suite $v<u$ sur la boule unité de $\ell^{1}(\mathbb{N})$.

Avant de donner la démonstration de ce théorème, signalons le problème ouvert suivant:

Problème. Comme dans le cas du second ordre (cf. [14] et [10]), est-il possible d'avoir un théorème de comparaison pour le problème de Cauchy équivalent?

Jusqu'a maintenant, nous avons une réponse positive dans le cas "borné" (voir [13]). Ce résultat a été montré à l'aide d'une formule du sous-différentiel du premier ordre de la somme de deux fonctions (cf. $[\mathbf{9}])$.

$\mathrm{Au}$ début, nous commencons par démontrer un cas particulier du Théorème 3.1:

Proposition 3.2. Soit $Y$ un espace de Banach vérifiant la condition (A). Soient $G: Y \times Y^{*} \longrightarrow \mathbb{R}$ un Hamiltonien uniformément continu, $w$ et $\widetilde{w}$ deux fonctions réelles sur $Y$, telles que $w(y) \leq K(1+\|y\|)$ et $\widetilde{w}(y) \geq-K(1+\|y\|)$ pour $y \in Y$ et une constante $K \geq 0$. 
Si w est une sous-solution de viscosité de

$$
w+G(y, D w)=0 \text { sur } Y
$$

et $\widetilde{w}$ est une sur-solution classique de (3.3), alors

$$
w \leq \widetilde{w} \text { sur } Y \text {. }
$$

Démonstration: La preuve est en deux étapes; dans la première nous montrons que $w-\widetilde{w}$ est bornée supérieurement sur $Y$, et dans le deuxième nous montrons le résultat désiré.

Première étape: Pour montrer que $w-\widetilde{w}$ est bornée supérieurement sur $Y$, nous aurons besoin du lemme suivant :

Lemme 3.3. Soit $Y$ un espace de Banach vérifiant la condition (A). Soit $f: Y \longrightarrow \mathbb{R} \cup\{-\infty\}$ une fonction semi-continue supérieurement et telle que $f(x) \leq M(1+\|x\|)$ pour $x \in Y$ et une constante $M \geq 0$. Si $f$ n'est pas bornée supérieurement, alors il existe $x_{m} \in Y, p_{m} \in \bar{D}^{+} f\left(x_{m}\right)$ tels que:

$$
\left\|p_{m}\right\| \text { est borné et } \lim _{m \rightarrow \infty} f\left(x_{m}\right)=+\infty \text {. }
$$

Démonstration: Considérons la fonction $a_{m}: \mathbb{R}^{+} \longrightarrow \mathbb{R}$ de classe $\mathcal{C}^{1}$, définie par

$$
a_{m}(t)= \begin{cases}0 & \text { si } 0 \leq t \leq m \\ \left((t-m)^{2}+1\right)^{\frac{1}{2}}-1 & \text { si } t>m\end{cases}
$$

Maintenant considérons la fonction $w_{m}: Y \longrightarrow \mathbb{R}$, définie par:

$$
w_{m}(x)=f(x)-M a_{m}(d(x)),
$$

où $d$ est la fonction de Leduc donnée par la Proposition 2.1. La fonction $w_{m}$ est semi-continue supérieurement. D'après le choix de la fonction $a_{m}, w_{m}$ est bornée supérieurement et le fait que $f$ n'est pas bornée supérieurement implique que $\sup \left\{w_{m}(x) ; x \in Y, m \in \mathbb{N}\right\}=+\infty$. Donc, d'après le Théorème 2.1 appliqué à $-f$, on peut trouver une fonction $g_{m}: Y \longrightarrow \mathbb{R}$ de classe $\mathcal{C}^{1}$ vérifiant:

(1) $\left\|g_{m}\right\|_{\infty}=\sup \left\{\left|g_{m}(x)\right| ; x \in Y\right\}<\frac{1}{m}$ et $\left\|g_{m}^{\prime}\right\|_{\infty}=\sup \left\{\left\|g_{m}^{\prime}(x)\right\|\right.$; $x \in Y\}<\frac{1}{m}$.

(2) $w_{m}-g_{m}$ atteint son maximum en un point $x_{m} \in Y$. 
D'où, si on pose $p_{m}=M a_{m}^{\prime}\left(d\left(x_{m}\right)\right) d^{\prime}\left(x_{m}\right)+g^{\prime}\left(x_{m}\right)$, on a $p_{m} \in D^{+} f\left(x_{m}\right)$. Et comme $a_{m}$ et $d$ sont Lipshitziennes, il existe une constante $N \geq$ 0 telle que $\left\|p_{m}\right\| \leq N$ pour $m \geq 1$. De plus, $f\left(x_{m}\right) \geq w_{m}\left(x_{m}\right) \geq$ $\sup \left\{w_{m}(x) ; x \in Y\right\}-\frac{1}{m}$, ce qui prouve que $\lim _{m \rightarrow \infty} f\left(x_{m}\right)=+\infty$.

Pour montrer la première étape, on suppose le contraire, donc la fonction $f=w-\widetilde{w}$, satisfait les conditions du Lemme 3.3; par conséquent il existe $x_{m} \in Y, p_{m} \in D^{+}(w-\widetilde{w})\left(x_{m}\right)$, tels que $\left\|p_{m}\right\|$ est borné et $\lim _{m \rightarrow \infty}(w-\widetilde{w})\left(x_{m}\right)=+\infty$. Mais $\widetilde{w}$ est une fonction de classe $\mathcal{C}^{1}$, ce qui donne $p_{m}+\widetilde{w}^{\prime}\left(x_{m}\right) \in D^{+} w\left(x_{m}\right)$.

La fonction $w$ est une sous-solution de viscosité de (3.3), donc

$$
w\left(x_{m}\right)+G\left(x_{m}, p_{m}+\widetilde{w}^{\prime}\left(x_{m}\right)\right) \leq 0 .
$$

La fonction $\widetilde{w}$ est une sur-solution de classique de (3.3), donc

$$
\widetilde{w}\left(x_{m}\right)+G\left(x_{m}, \widetilde{w}^{\prime}\left(x_{m}\right)\right) \geq 0
$$

Par conséquent,

$$
w\left(x_{m}\right)-\widetilde{w}\left(x_{m}\right) \leq G\left(x_{m}, \widetilde{w}^{\prime}\left(x_{m}\right)\right)-G\left(x_{m}, p_{m}+\widetilde{w}^{\prime}\left(x_{m}\right)\right) .
$$

Comme $G$ est uniformément continue et $\left\|p_{m}\right\|$ est borné, en utilisant le Lemme 3.4 ci dessous, on obtient une contradiction avec (3.4).

Deuxième étape: Fixons $\varepsilon>0$. La fonction $w-\widetilde{w}$ est semi-continue supérieurement et d'après la première étape, elle est bornée supérieurement. D'après le Théorème 2.1 appliqué à $f=-(w-\widetilde{w})$, il existe $x_{0} \in Y$ et $p \in D^{+}(w-\widetilde{w})\left(x_{0}\right)$, tels que $\|p\|<\varepsilon$ et $(w-\widetilde{w})\left(x_{0}\right)>\sup (w-\widetilde{w})-\varepsilon$.

La fonction $w$ est une sous-solution de viscosité de (3.3), donc

$$
w\left(x_{0}\right)+G\left(x_{0}, p+\widetilde{w}^{\prime}\left(x_{0}\right)\right) \leq 0 .
$$

La fonction $\widetilde{w}$ est une sur-solution classique de (3.3), donc

$$
\widetilde{w}\left(x_{0}\right)+G\left(x_{0}, \widetilde{w}^{\prime}\left(x_{0}\right)\right) \geq 0 .
$$

Par conséquent,

$$
\begin{aligned}
\sup (w-\widetilde{w}) & \leq(w-\widetilde{w})\left(x_{0}\right)+\varepsilon \\
& \leq G\left(x_{0}, \widetilde{w}^{\prime}\left(w_{0}\right)\right)-G\left(x_{0}, p+\widetilde{w}^{\prime}\left(w_{0}\right)\right)+\varepsilon .
\end{aligned}
$$

De plus, $\|p\| \leq \varepsilon$, et $G$ est uniformément continue, donc en faisant tendre $\varepsilon$ vers zéro, on obtient:

$$
\sup _{Y}(w-\widetilde{w}) \leq 0
$$


Démonstration du Théorème 3.1: Soit $\varepsilon>0$ fixe. Considérons l'espace de Banach $Y=X \times X$, qui satisfait l'hypothèse (A) (il suffit de prendre $B(x, y)=b(x) b(y))$. Le Hamiltonien $G$ défini sur $Y \times Y^{*}$ par $G(x, y, p, q)=H_{1}(x, p)-H_{2}(y,-q)$ est uniformément continu. Nous affirmons qu'il existe $\widetilde{K}_{\varepsilon} \geq 0$ telle que la fonction

$$
\widetilde{w}(x, y)=\left(\varepsilon^{2}+\widetilde{K}^{2} d(x-y)^{2}\right)^{\frac{1}{2}}+\sup _{X \times X^{*}}\left(H_{1}-H_{2}\right)
$$

est une sur-solution classique de (3.3), où $d$ est la fonction de Leduc donnée par la Proposition 2.1.

Pour cette affirmation, nous avons besoin du lemme suivant:

Lemme 3.4. Soient $X$ un espace de Banach et $v: X \longrightarrow \mathbb{R}$ une fonction uniformément continue. Alors, pour tout $\varepsilon>0$, il existe un nombre réel $K_{\varepsilon}>0$ tel que:

$$
|v(x)-v(y)| \leq \varepsilon+K_{\varepsilon}\|x-y\|
$$

pour $x, y \in X$.

Démonstration du Lemme 3.4: Soit $\varepsilon>0$ fixé. Comme $v$ est uniformément continue sur $X$, il existe $\delta_{\varepsilon}>0$ tel que $|v(x)-v(y)| \leq \varepsilon$ dès que $\|x-y\| \leq \delta_{\varepsilon}$. Soient $x$ et $y$ deux éléments de $X$. Si $\|x-y\| \leq \delta_{\varepsilon}$, on a le résultat. Sinon il existe $n \in \mathbb{N}^{*}$ tel que $n+1>\frac{\|x-y\|}{\delta} \geq n$. Soit $\left(x_{k}\right)_{k=0}^{n+1}$ une suite finie d'éléments de $X$ telle que:

$$
\left\{\begin{array}{l}
x_{k}=x+\frac{k \delta}{\|x-y\|}(y-x) \text { pour } k=0,1, \ldots, n \\
x_{n+1}=y
\end{array}\right.
$$

On a $\left\|x_{k+1}-x_{k}\right\|=\delta_{\varepsilon}$ pour $k=0,1, \ldots, n-1$; de plus

$$
y-x_{n}=(y-x)\left(1-n \frac{\delta_{\varepsilon}}{\|x-y\|}\right),
$$

et donc $\left\|y-x_{n}\right\|=\|y-x\|-n \delta_{\varepsilon}<\delta_{\varepsilon}$. Par conséquent,

$$
\begin{aligned}
|v(x)-v(y)|=\left|\sum_{k=0}^{n} v\left(x_{k}\right)-v\left(x_{k+1}\right)\right| & \leq \sum_{k=0}^{n} \mid v\left(x_{k}\right)-v\left(x_{k+1} \mid\right. \\
& \leq(n+1) \varepsilon=\varepsilon+n \varepsilon \\
& \leq \varepsilon+\frac{\varepsilon}{\delta_{\varepsilon}}\|x-y\|
\end{aligned}
$$


Il suffit donc de prendre $K_{\varepsilon}=\frac{\varepsilon}{\delta_{\varepsilon}}$.

Appliquons ce lemme à $f=H_{2}$ sur l'espace de Banach $Y=X \times X^{*}$, il existe $K_{\frac{\varepsilon}{2}} \geq 0$ tel que $\left|H_{2}(x, p)-H_{2}(y, q)\right| \leq \frac{\varepsilon}{2}+K_{\frac{\varepsilon}{2}}(\|x-y\|+\|p-q\|)$ pour $x, y \in X$ et $p, q \in X^{*}$.

D'où

$$
\begin{aligned}
\widetilde{w}(x, y) & +G\left(x, y, D_{x} \widetilde{w}(x, y), D_{y} \widetilde{w}(x, y)\right) \\
& =\left(\varepsilon^{2}+\widetilde{K}^{2} d(x-y)^{2}\right)^{\frac{1}{2}}+\sup _{X \times X^{*}}\left(H_{1}-H_{2}\right) \\
& +H_{1}\left(x, D_{x} \widetilde{w}(x, y)\right)-H_{2}\left(y,-D_{y} \widetilde{w}(x, y)\right) \\
& \geq\left(\varepsilon^{2}+\widetilde{K}^{2} d(x-y)^{2}\right)^{\frac{1}{2}}-\frac{\varepsilon}{2}-K_{\frac{\varepsilon}{2}}\|x-y\| .
\end{aligned}
$$

Si on choisit $\widetilde{K} \geq 2 K_{\frac{\varepsilon}{2}}^{2}$, il est facile de voir que la dernière quantité est positive.

Finalement, la fonction $w(x, y)=u(x)-v(y)$ est une sous-solution de viscosité de (3.3). De plus comme $\|(x, y)\|=\|x\|+\|y\|$ est une norme équivalente $\operatorname{sur} X \times X$, on a $w(x, y) \leq C(1+\|(x, y)\|)$ et $\widetilde{w}(x, y) \geq$ $-C(1+\|(x, y)\|)$. Donc, d'après la Proposition 3.2, $w \leq \widetilde{w} \operatorname{sur} X \times X$, ce qui complète la preuve.

\section{Existence et régularité de la solution de viscosité}

Rappelons que si $u$ est une fonction localement bornée définie sur $X$, l'enveloppe semi-continue supérieure $u^{*}$ de $u$ est définie par:

$$
u^{*}(x)=\varlimsup_{r \rightarrow 0}\{u(y) ;\|y-x\| \leq r\}
$$

et l'enveloppe semi-continue inférieure $u_{*}$ de $u$ est définie par:

$$
u_{*}(x)=\varlimsup_{r \rightarrow 0}\{u(y) ;\|y-x\| \leq r\} .
$$

Dans cette section, nous utilisons le principe fondamental suivant appelé méthode de Perron pour les solutions de viscosité, qui a été prouvé par H. Ishii (cf. [19]). Nous renvoyons à [8, Proposition 3.4] pour la preuve de l'enoncé suivant:

Théorème. Supposons que l'espace de Banach $X$ vérifie l'hypothèse $(A)$ et le Hamiltonien $H$ défini sur $X \times X^{*}$ est continu. Soient $f$ une sous-solution de viscosité de (1.1) et $g$ une sur-solution de viscosité de (1.1). Supposons que $f \leq g$ sur $X$. Alors, il existe une fonction $u$ définie sur $X$, telle que $f \leq u \leq g$, $u^{*}$ est une sous-solution de viscosité de (1.1) et $u_{*}$ est une sur-solution de (1.1).

Notre résultat est le suivant: 
Théorème 4.1. Supposons que l'espace de Banach $X$ satisfait la condition (A). Soit $H: X \times X^{*} \longrightarrow \mathbb{R}$ un Hamiltonien uniformément continu. Alors le problème (1.1) a une unique solution de viscosité u verifiant: il existe une constante $C$ telle que pour tout $x \in X,|u(x)| \leq$ $C(1+\|x\|)$. De plus, cette solution est uniformément continue sur $X$.

\section{Démonstration:}

Comme $H$ est uniformément continue sur $X \times X^{*}$, d'après le Lemme 3.4, il est facile de voir qu'il existe $M \geq 0$ tel que $f(x)=-M(1+$ $\|x\|)$ est une sous-solution de viscosité de $(1.1)$ et $g(x)=M(1+\|x\|)$ est une sur-solution de viscosité de (1.1). Maintenant, en utilisant la méthode de Perron pour les solutions de viscosité, on peut trouver une fonction $u: X \longrightarrow \mathbb{R}, f \leq u \leq g$ telle que $u^{*}$ est une sous-solution de viscosité de (1.1) et $u_{*}$ est une sur-solution de viscosité de (1.1). Appliquons le Théorème 3.1 : on obtient $u^{*} \leq u_{*}$, et puisque par définition $u_{*} \leq u^{*}$, on conclut que $u^{*}=u_{*}=u$.

Pour avoir un module de continuité de la solution, on fixe $h \in X$ et on définit deux Hamiltoniens $H_{1}$ et $H_{2}$ en posant $H_{1}(x, p)=H(x, p)$ et $H_{2}(x, p)=H(x+h, p)$. Soient $u_{1}$ la solution de viscosité sous linéaire de $u+H_{1}(x, D u)=0$ et $u_{2}$ la solution de viscosité sous linéaire de $u+H_{2}(x, D u)=0$. Du Théorème 3.2 , on en déduit l'inégalité suivante:

$$
\left|u_{1}(x)-u_{2}(x)\right| \leq \sup _{X \times X^{*}}|H(x+h, p)-H(x, p)| \text { pour } x \in X .
$$

Mais $H$ est uniformément continue, donc pour tout $\varepsilon>0$, il existe $\delta_{\varepsilon}$ tel que pour chaque $h \in X$ et $p \in X^{*}$, on a $\|h\|<\delta$ implique $|H(x+h, p)-H(x, p)|<\varepsilon$. Soit $u$ la solution de viscosité de (1.1). Il est clair que $u_{1}(x)=u(x)$ et $u_{2}(x)=u(x+h)$ pour $x \in X$. Par conséquent, $\sup _{\|h\|<\delta}|u(x)-u(x+h)|<\varepsilon$, ce qui montre que $u$ est uniformément continue sur $X$ et que la fonction réelle et positive $\omega$ définie par $\omega(s)=\sup _{\|h\|<s}|H(x, p)-H(x+h, p)|$ pour $s \geq 0$ est un module de continuité de la solution $u$.

Je tiens à remercier Robert Deville pour ses conseils et ses précieuses suggestions.

\section{Références}

1. G. BARles, Remarques sur des résultats d'existence pour les équations de Hamilton-Jacobi du premier ordre, Ann. Inst. H. Poincaré. Anal. Non linéare. 2 (1985), 21-33. 
2. J. M. BorweIn AND D. Preiss, A smooth variational principle with applications to subdifferentiability and to differentiability of convex functions, Trans. Amer. Math. Soc. 303 (1987), 517-527.

3. I. Capuzzo Dolcetta, On a discrete approximation of the Hamilton-Jacobi equation of dynamic programming, Appl. Math. Optim. 10 (1983), 367-377.

4. M. G. Crandall. L. C. Evans and P .L. Lions, Some properties of viscosity solutions Hamilton-Jacobi equations, Trans. Amer. Math. Soc. 282 (1984), 487-502.

5. M. G. Crandall and P. L. Lions, Condition d'unicité pour les solutions généralisées des équations de Hamilton-Jacobi du premier ordre, C. R. Acad. Sci. Paris. 292 (1981), 183-186.

6. M. G. Crandall and P. L. Lions, Viscosity solutions of Hamilton-Jacobi equations, Trans. Amer. Math. Soc. 277 (1983), 1-42.

7. M. G. Crandall and P. L. Lions, Hamilton-Jacobi equations in infinite dimensions, Part I: Uniqueness of viscosity solutions, $J$. Funct. Anal. 62 (1985), 379-396; Part II: Existence of viscosity solutions, 65 (1986), 368-405; Part III, 68 (1986), 214-247; Part IV: Unbounded linear terms, 90 (1990), 237-283; Part V: B-continuous solutions, 97 (1991), 417-465; Part VI: Nonlinear $A$ and Tataru's method refined; Part VII: The $H J B$ equation is not always satisfied.

8. R. Deville, Stability of subdifferentials of non convex functions in Banach spaces, to appear in Set-Valued Analysis.

9. R. Deville and E. M. EL Haddad, The subdifferential of the sum of two functions in Banach spaces, I. First order case, preprint.

10. R. Deville and E. M. EL Haddad, The subdifferential of the sum of two functions in Banach spaces, II. Second order case, Bull. Austr. Math. Soc. 51 (1995), 235-248.

11. R. Deville, G. Godefroy and V. Zizler, A smooth variational principle with applications to Hamilton-Jacobi equations in infinite dimensions, J. Funct. Anal. 111 (1993), 197-212.

12. R. Deville, G. Godefroy And V. Zizler, "Smoothness and renormings in Banach spaces," Pitman Monographs in Mathematics 64, Longman Scientific and Technical, 1993.

13. E. M. EL HADDAD, Viscosity solutions of Hamilton-Jacobi equations in infinite dimensions, preprint.

14. E. M. EL HADDAD, Calcul sous-différentiel et solutions de viscosité des équations de Hamilton-Jacobi, Thèse de Doctorat de Mathématiques de l'Université Bordeaux 1 (1994).

15. R. G. Haydon, A conterexample to several questions about scat- 
tered compact spaces, Bull. London Math. Soc. 22 (1990), 261-268.

16. H. IsHII, Uniqueness of unbounded viscosity solutions of Hamilton-Jacobi equations, Indiana Univ. Math. J. 33 (1984), 721-748.

17. H. IsHII, Remarks on existence of viscosity solutions of Hamilton-Jacobi equations, Bull. Fac. Sci. Eng. Chuo Univ. 26 (1986), 214-247.

18. H. IsHII, Existence and uniqueness of solutions of Hamilton-Jacobi equations, Funkcial. Ekvac. 29 (1986), 167-188.

19. H. IsHiI, Perron's method for Hamilton-Jacobi equations, Duke Math. J. 55 (1987), 369-384.

20. H. IsHII, Representation of solutions of Hamilton-Jacobi equations, Non. Anal. Theor. Meth. Appl. 12 (1988), 121-141.

21. C. Stegall, Optimization of functions on certain subsets of Banach spaces, Maths. Ann. 236 (1978), 171-176.

\author{
Université de Franche-Comté \\ Laboratoire de Mathématiques \\ Faculté des Sciences \\ Route de Gray \\ 25000 Besançon \\ FRANCE
}

Rebut el 22 de Desembre de 1994 